\title{
Spontaneous Rupture of Renal Artery Aneurysm into the Renal Pelvis: An Unusual Bause of Gross Hematuria and Urinary Retention
}

Hmida Wissem, Bouassida Khaireddine, Jaidane Mehdi, Ben Soba Nebil, Mosbah Ali Tahar

From the Department of Urology, Hospital of Sahloul, Sousse, Tunisia.

\section{Abstract:}

Aneurysm of the renal artery is a rare condition with relatively uncommon symptoms. A 58-year-old male patient presented to the emergency department with urinary retention caused by massive hematuria. He was hemodynamically stable, and Ultrasound examination of the abdomen showed a massive clot of blood in the bladder with no signs of tumor. Multi-detector CT images in the arterial phases showed bleeding in the left renal pelvis and an intra-renal lower pole aneurysm. The CT findings were consistent with a rupture of the aneurysm into the left renal pelvis. The aneurysm was embolized with a coil. Immediate post-interventional check-up injections revealed no sign of renal aneurysm. One month later, clinical follow up revealed no hematuria.

Key words: Aneurysm, Urinary Retention, Aneurysm Ruptured, Embolization, Hematuria, Neoplasms.

\section{Introduction}

Renal Artery Aneurysm (RAA) is often reported to be uncommon, occurs in about $0.09 \%$ to $0.3 \%$ of individuals [1]. Recent advances in angiography and vascular CT have, however, shown that the prevalence of this disease is rising on a global scale. In fact, the symptoms related to aneurysm are uncommon, which makes the clinical diagnosis of renal artery aneurysm extremely difficult [2]. The etiology of renal artery aneurysms may be idiopathic, mycotic or traumatic with fibromuscular dysplasia being a predisposing factor [3].

The present paper reports a rupture of renal artery aneurysm into the renal pelvis which presented as acute urinary retention due to blood clots caused by massive hematuria. So far, and to the authors' knowledge, no previous report has been published on the occurrence of this mode of disclosure.

\section{Case Report}

A 58-year-old man presented to the emergency department with acute urinary retention. His past medical history revealed no abnormalities. The patient was afebrile, with normal blood pressure $(130 / 80 \mathrm{~mm} \mathrm{Hg})$

Corresponding Author: Dr Bouassida Khaireddine

Email: khayri14@hotmail.com

Received: May 19, 2013 | Accepted: May 21, 2013 | Published Online: June 5, 2013

This is an Open Access article distributed under the terms of the Creative Commons Attribution License (creativecommons.org/licenses/by/3.0)

Conflict of interest: None declared | Source of funding: Nil | DOl: http://dx.doi.org/10.17659/01.2013.0039 
and an average heartbeat of 77 beats per minute. Abdominal examination showed a distended bladder. Creatinine levels were normal, and hemoglobin was $13 \mathrm{~g} / \mathrm{dl}$. Transurethral catheter was put in place, showing massive hematuria with clots.

Ultrasound examination of the abdomen showed a massive blood clot in the bladder without tumor and left renal cyst in the upper pole [Fig.1]. The patient was hemodynamically stable and was transferred to the Urology department.

Cystoscopy showed the same massive clot of blood without tumor. Bilateral ureteroscopy showed bleeding from the left kidney, with no identified causes, and a double-J stent was, therefore, placed for drainage. A multi-detector CT in the arterial phases showed bleeding in the left renal pelvis and an intra renal lower pole aneurysm. The CT findings were consistent with a rupture of the aneurysm into the left renal pelvis [Fig. 2,3]. The CT images also showed an aneurysm in the medium hepatic artery and an aneurysmal dissection in the celiac trunk [Fig. 3,4].

Etiological research of aneurysms showed no underlying vasculitis. The search for ANCA (antineutrophil cytoplasmic antibodies) against small and medium-size blood vessels was negative, and the Wegener's granulomatosis and Churg Strauss syndrome was, therefore, eliminated.

The patient was transferred for interventional radiology and vascular surgery evaluation. The aneurysm was embolized by means of a coil. Immediate post-interventional check-up injections revealed no signs of renal aneurysm [Fig. 5,6]. One month later, clinical follow-up revealed no hematuria.

\section{Discussion}

Renal artery aneurysms (RAA) accounts for $10-22 \%$ of all visceral aneurysms, with $25 \%$ of RAA being intraparenchymal. They are bilateral in 19\% of cases and multiple in 32\% [4]. The typical age of onset for the disease is $40-60$ years. It has no gender predilection, though RAA rupture is more common in women at the reproductive age [5].

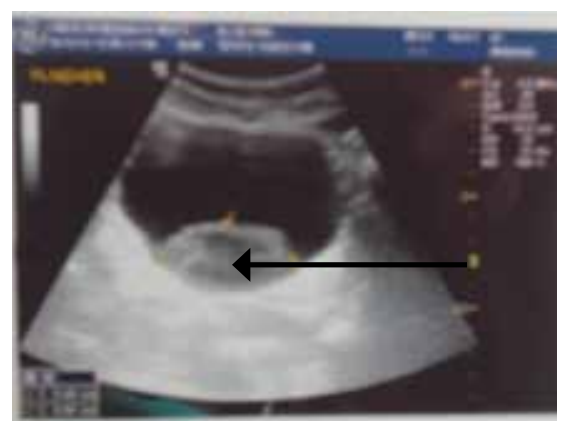

Fig. 1: Abdominal Ultrasound showing a clot of blood in the bladder 


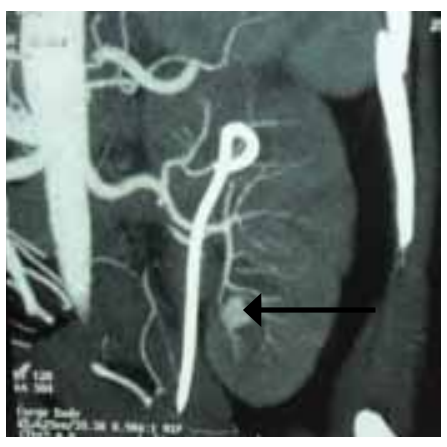

Fig.2: Coronal reconstructed CT image: Aneurysm is located in the lower pole of the left kidney adjacent to renal pelvis.

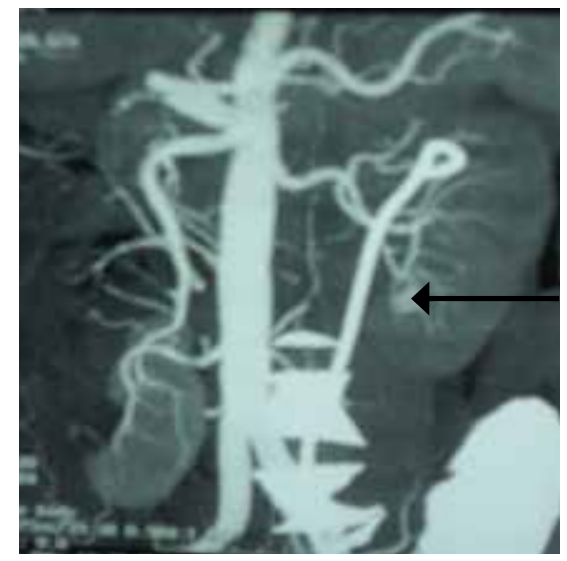

Fig.3: Coronal reconstructed CT image. Dissecant aneurysm in the coeliac trunk (white arrow) and a renal artery aneurysm (black arrow).

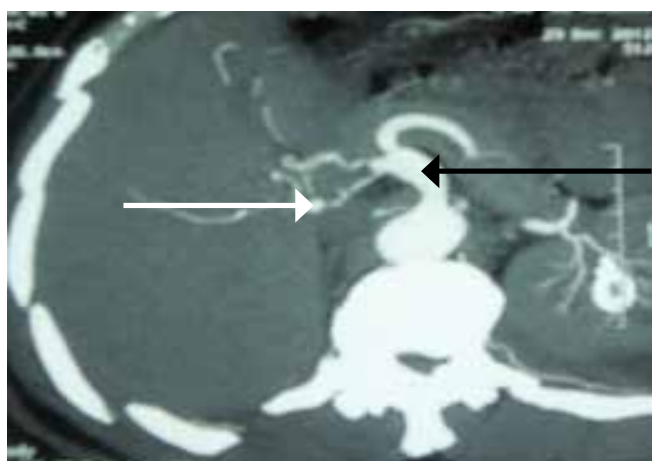

Fig.4: Transverse CT images shows dissecant aneurysm in the coeliac trunk (black arrow) and little aneurysm of the middle hepatic artery (white arrow). 


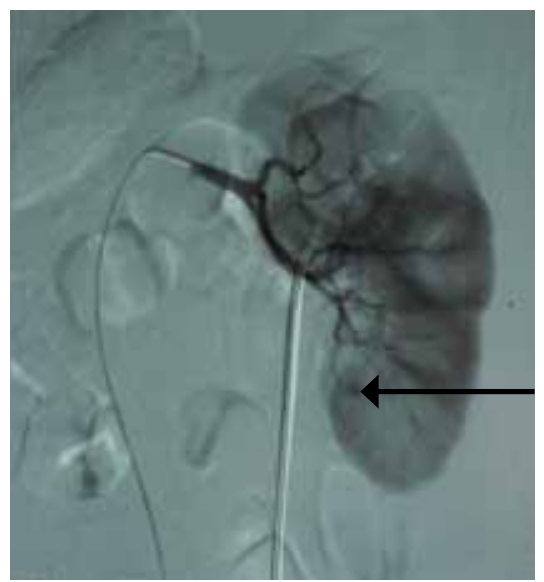

Fig.5: Digital angiography showing an aneurysm in the lower pole of the left kidney.

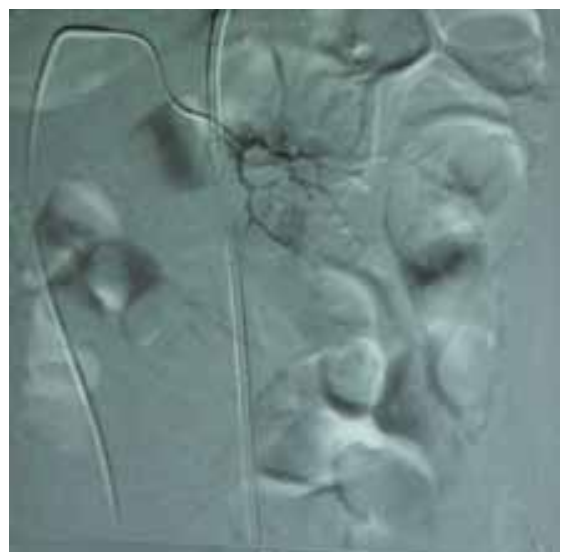

Fig.6: Post-interventional check-up injections that revealed no signs of aneurysm.

Clinical findings of renal artery aneurysm are non-specific, including microscopic hematuria, hypertension and flunk pain [2]. The etiology of renal artery aneurysms may be idiopathic, mycotic or traumatic but, in most cases, there is an underlying diffuse systemic disease. Predisposing factors include arteriosclerosis, fibromuscular dysplasia, congenital malformations of the kidney, renal angiomyolipoma, pregnancy, and trauma [6]. The risk of rupture increases during pregnancy and in patients with polyarteritis nodosa [5].

With the use of MDCT, the renal vasculature can be demonstrated, and abnormalities become easier to depict. In fact, MDCT offers unique capabilities for diagnosis and follow-up of renal artery aneurysm. It also gives a good overview of anatomical topographical relationships. Reconstructed coronal CT images exquisitely demonstrate the presence and rupture of the aneurysm in the pelvis and in the ureter $[2,4]$.

Indications for surgery include hypertension with renal failure, hypertension with a solitary kidney, pain, hematuria, enlarging aneurysm, and aneurysm diameter of more than $2 \mathrm{~cm}$ [2]. Rupture of RAA is more common in women at the reproductive age. Instances of atraumatic intraparenchymal RAA, such as our case, are usually 
caused by fibromuscular dysplasia [7]. Most cases are discovered incidentally or during the evaluation for renovascular hypertension $[4,5]$.

RAA was previously managed using surgical therapy (nephrectomy, revascularisation) or catheter-directed embolization. Recently, the application of percutaneous intervention with stent graft has been introduced [8]. Trans-arterial embolization was first described in 1973 by Bookstein and Goldstein, and, nowadays, it is being performed as first-line treatment for aneurysms, such as the case of our patient, with good results $[9,10]$.

\section{Conclusion}

Renal artery aneurysms rupture in renal pelvis is rare. It can cause massive hematuria with urinary retention due to blood clots. This diagnosis should be considered especially when Multi-detector CT demonstrates the presence or rupture of the aneurysm in the pelvis. Sometimes the rupture can cause hypotension and shock. Coverage should, therefore, be early, which, unfortunately, was not the case with our patient. The endovascular approach is a good alternative to invasive surgical procedures.

\section{References}

1. Castillo OA, Vitagliano GJ, Sanchez-Salas R, Chamorro H, Fava M, Díaz MA. Laparoscopic repair of renal artery aneurysm: a description of the technique and results in 2 cases. Surg Laparosc Endosc Percutan Tech. 2008; 18:379-383.

2. De Wilde V, Devue K, Vandenbroucke F, Breucq C, De Maeseneer M, De Mey J. Rupture of renal artery aneurysm into the renal pelvis, clinically mimicking renal colic: diagnosis with multidetector $\mathrm{CT}$. $\mathrm{Br} \mathrm{J}$ Radiol. 2007;80:262-264.

3. Gümüștaș $S$, Ciftçi E, Bircan Z. Renal artery aneurysm in a hypertensive child treated by percutaneous coil embolization. Pediatr Radiol. 2010;40:1 285-1287.

4. Henke PK, Cardneau JD, Welling TH III, Upchurch GR Jr, Wakefield TW, Jacobs LA et al. Renal artery aneurysms: a 35-year clinical experience with 252 aneurysms in 168 patients. Ann Surg. 2001;234:454463.

5. Fraser GE, Poncia $\mathrm{H}$. Spontaneous renal artery aneurysm rupture: an unusual cause of abdominal pain and syncope. Emerg Med J. 2009;26:619-620.

6. Marwah S, Singla S, Kalra R, Marwah N, Singh SP. Is it renal colic or ruptured dissecting aneurysm of renal artery? A case report. Cases J. 2009;2:9398.

7. Cheng C, Jenkins IL, Ramsay JWA and Hendry WF. Spontaneous rupture of renal artery aneurysm. J R Soc Med. 1990;83:464-465.

8. Aranzulla TC, Colombo A, Sangiorgi GM. Successful endovascular renal artery aneurysm exclusion using the Venture catheter and covered stent implantation: a case report and review of the literature. J Invas Cardiol. 2007; 19:246-253.

9. Yakup Y, Bora P, Barbaros C, Bozkurt G, Burak D, Cenk BY. Endovascular management of iatrogenic renal artery aneurysm and arteriovenous fistula. Saudi J Kidney Dis Transpl. 201 2;23:838-840.

10. Nagahara A, Nishimura K, Okuyama A. A giant idiopathic renal arteriovenous fistula associated with high-output heart failure. Int J Urol. 2009;1 6:648-649. 